\title{
IL REGISTRO DELLA CONFRATERNITA DEI PELLICCIAI DI UDINE. A cura di Federico Vicario. Biblioteca di Lingua e Letteratura friulana, 4. - Forum, Editrice Universitaria Udinese, Udine 2003; pp. 204.
}

L'edizione, in una veste tecnicamente impeccabile, ha come scopo principale quello di presentare il registro di una importante confraternita udinese del tardo Medioevo, quella dei pellicciai. In questo ambiente climatico tale mestiere deve essere stato importante; e vedrei una prova nel fatto che viene menzionato, a parte gli beçars - macelatores (es. 42), l'unico, negli Esercizi di versione dal volgare friulano in latino nel sec. XIV in una scuola notarile cividalese dove si legge: L'ager galt, qual è d'estat, fas spargna lis pliçis, chosa ricrisint agl piliças. Ager calidus, qualis est in estate, facit parsimonucare renones, re cuius tedet pelixarios, (es. 40). Il curatore della pubblicazione del Registro, Federico Vicario, vanta già una decina di pubblicazioni di testi analoghi e con l'attuale ha aggiunto un ulteriore testo prezioso per la nostra conoscenza del friulano antico. Il Registro è l'elenco dei censuari, però, oltre ad essere un documento della vita e dei rapporti sociali del Tre- e Quattrocento, offre un quadro linguistico del friulano in un'epoca in cui la letteratura vera e propria in friulano offre poco.

Il Registro medievale pubblicato occupa, comprese le esaurienti annotazioni e note, le pagine 37-171. Lo precedono le note introduttive, dove troviamo presentati i criteri dell'edizione. Il Registro è seguito dalla Appendice con il Glossario, pp. 173-184, dall'Indice onomastico, pp. 185-194, e dall'Indice degli argomenti, 195-196; non fa meraviglia il fatto che la stragrande maggioranza di questi appartenga a fenomeni fonetici. Chiude l'opera una selezionata ma esauriente bibliografia, pp. 197-204. Il Registro consiste in 46 carte, recto/verso; il testo è abbondantemente commentato nelle preziose note le quali, oltre ad una accurata traduzione italiana, offrono un ricco e dettagliato quadro linguistico ed extralinguistico, annotazioni storiche, soprattutto. Non sorprende che le note riguardino per lo più il vocabolario e la semantica dei vocaboli usati nel testo, compresi antroponimi e toponimi, oltre alla veste fonica che possiamo scoprire attraverso la grafia, benché a volte oscillante; ci sia permesso di aggiungere in lode del curatore che non è trascurato nemmeno il lato sintattico. Per quanto riguarda il verbo troviamo confermata l'esistenza del preterito semplice: una chiassa la quale el comperà, carta $2 \mathrm{r}$, la qual lasà la Gundulina, carta $2 \mathrm{r}$, ecc. A volte si può vedere nell'impiego della forma semplice l'influenza dell'uso della corrispondente formula notarile in latino: nella carta 6v si legge infatti Ego... notarius ordinarius scripsi ... Et io Piero Pollame lo copiai ... di mia man propria; un analogo influsso latino sarà da vedere senz'altro nell'uso del preterito semplice in Nicolau filg chu fuo di /.../ Margaretta mogli chu fuo di..., carta 1r. La formula latineggiante si ripete in molti altri casi, non solo in questa frase fissa; troviamo il preterito semplice dello stesso verbo di essenza o esistenza in sta in borgo di Glemona in lis casis che forin di Fantùs, c 42v. Per la pura morfologia del sostantivo si constata che 
il plurale non è sempre sigmatico e nel campo della morfosintassi è inoltre sottolineata con molta cura la mancanza di accordo tra vari elementi, ad es. dinars $x x l i$ quali forin lasat, $\mathrm{c} 30 \mathrm{v}$. Altrove il mancato accordo riguarda l'erroneo uso del numero grammaticale: $d$-una part posset gl-areç di Denel rodar, c. $3 \mathrm{v}$. Il curatore segnala scrupolosamente queste dissonanze che non potrebbero essere attribuite solo a una trascurata grafia. Inoltre, il pronome atono non si aggiunge alla forma tonica del pronome o a un sostantivo il che è una caratteristica tipica del soggetto nel friulano di oggi. La ripresa pronominale è quella arcinota in friulano e in romanzo in generale. Qualche cenno si trova per il pronome relativo e per la subordinata relativa. L'editore giustamente corregge la troppo frettolosa opinione che l'impiego del pronome relativo esplicito il quale sia un latinismo, che si trova, ad. es., negli EsERCIZI, testé menzionati, di uso scolastico dei futuri notai alla scuola di Cividale. E' però ovvio che la sintassi, vale a dire soprattutto la costruzione della frase, rimane povera, come del resto c'era da aspettarsi. Un'analisi analoga dei testi antichi veneziani di Alfredo Stussi dimostra la medesima tendenza a una disperata monotonia. Si tratta, insomma, di un registro.

Il fulcro di tutto l'enorme lavoro di Vicario, ovviamente, sta nell'aver raccolto una miniera di vocaboli che interessano il lessico e con questo anche la semantica. Da una parte abbiamo una ricca raccolta dei nomi di mestieri. Se gli iscritti della confraternita furono piliçars/pilliçars, c. 3r, i loro padri e i loro vicini erano di mestieri differenti. Così troviamo: caligier/caligar/cialar/chaliar, cortelar, fari, lauar, mulinar, sartor, scarpar, spadar, stringai, taschiar, temesar, ecc. Si merita un particolar cenno fornedor, non fosse che per la corrispondente forma femminile: fornedressa, c. $2 \mathrm{r}$.

Il Glossario racchiude tutti i vocaboli presenti nel testo, anche quelli delle note alle singole carte, riordinati secondo la loro provenienza: vi si trovano oltre il friulano parecchie lingue che hanno arricchito il lessico del Registro e perciò del friulano dell'epoca, magari solo con qualche elemento lessicale. E' da notare che alcune tra le lingue, e soprattutto quelle delle fasi antiche, come il germanico o l'antico alto tedesco appaiono soprattutto come elementi degli antroponimi, più raramente dei toponimi. Non sorprendono alcuni toponimi del gallico, come Broyli, nell'antico francese breuil, oppure Grevar, Nimis che, in qualche modo, si conservano ancora fino ad oggi in francese, cfr. grève, Nimes, e ugualmente il germ. kasto casto, c. 40r, frl. 'ciast' o la longobarda braida. Più sorprendente è il termine longobardo fanthio che si conserva in Domeni Chiandit Fant, c. 11v - Vicario ammette la possibilità di farlo derivare non dal lat. INFANTEm, bensì dal menzionato sostantivo longobardo.

$\mathrm{Ci}$ attira in particolar modo l'apporto sloveno (Vicario scrive sempre correttamente "sloveno", non genericamente slavo). Tra i termini di provenienza slovena il più vetusto è senz'altro il noto slavismo pustota; qui, è degno di nota, perché si trova in una frase in latino: duo alia /.../ sunt in pustota 'due altri possedimenti sono in- 
colti', c. $42 \mathrm{v}$. La traduzione del curatore è sicuramente corretta, anzi, il termine sloveno, in origine senz'altro un sostantivo, forma con la preposizione una specie di avverbio, il che assicura che il vocabolo è diventato patrimonio della lingua ricevente. Nel toponimo Loca in Gl-areç di Pieri da Locha 'gli eredi di Pietro di Lonca', c. 25r, è interessante constatare la denasalizzazione della vocale accentata: il toponimo, perciò, sembra essere relativamente recente, vale a dire, dopo l'VIII secolo. Sono, di certo, di provenienza slovena i due antroponimi/cognomi Bellem e Cirnich che nascondono due colori, rispettivamente bel 'bianco' e crn 'nero'. Così anche Guirch in Denel di Guirch 'Daniele di Virco', c. 3v. Il toponimo, dall'autore correttamente interpretato con lo slov. vir 'fontana' e non forse da vrh 'cima, altura', è frequente nella geonomastica slovena. Forse più problematici sono Sgubiriça, c. 6v dallo sl. guba e fradagla, c. 2r; noto quest'ultimo nello sloveno carsico - l'autore cita il saggio della compianta Neva Godini, CE FASTU?, LVII (1981) - però senza dubbio di provenienza romanza, vale a dire friulana. La conoscenza dello sloveno da parte dell'autore è degna di ogni lode. E soprattutto, è un fatto che ho ammirato già nei lavori di Tagliavini, di Pellegrini, di Francescato, di Doria, di Frau per menzionare solo a me i più noti. Giacché, in alcune opere, lo sloveno è come se non esistesse: per dare un esempio, è scientificamente triste vedere il termine di otava 'fieno di secondo taglio' in un vocabolario giuliano, inserito s. v. otto il che toglie a un tale vocabolario molto del suo valore scientifico. Ci volle per correggere l'errore l'autorità di Mario Doria, v. il suo Grande dizionario del dialetto triestino: "Dallo sloveno otâva, dal verbo otáviti 'ristorare, rifocillare'. Il lat. octavus non c'entra dunque affatto."

L'opera di Federico Vicario è frutto di un lavoro assiduo e di ampia competenza linguistica. $\mathrm{E}^{\prime}$ inoltre una raccolta di prima mano con un ampio e sicuro commentario linguistico. Per un periodo che per il friulano non conosce una vasta produzione letteraria, una tale opera è doppiamente preziosa. E' ovviamente interessante per la storia culturale e sociale del tardo Medio evo; al linguista, e non solo agli specialisti per la lingua friulana, offre una quantità d'informazioni genuine che aiutano a crearsi l'immagine linguistica del friulano come risulta da un testo, venato dalle precedenti o coeve esibizioni notarili latine, in un periodo in cui il linguaggio settoriale, nel nostro caso quello dei pellicciai, del tardo Medio evo mostra una stabile indipendenza e autonomia.

Mitja Skubic 